\title{
Applicability of The Resources of Behavioral Medicine in Dental Anxiety
}

\author{
Okuhara Monica ${ }^{1}$, Colas Osmar ${ }^{2}$, Martins Maria Benedicta Al ${ }^{1,2}$, Gonçalves Aurora ${ }^{1,2}$, Mello Paulo ${ }^{2}$, Monson Carlos ${ }^{1 *}$, \\ Leite José Roberto ${ }^{2}$ and Petri Valeria ${ }^{1}$
}

${ }^{1}$ Department of Dermatology, Paulista School of Medicine, Federal University of Sao Paulo, UNIFESP, Brazil

${ }^{2}$ Department of Psychobiology, Paulista School of Medicine, Federal University of Sao Paulo, UNIFESP, Brazil

*Corresponding author: Monson Carlos, Department of Dermatology, Paulista School of Medicine, Federal University of Sao Paulo, UNIFESP, Brazil

\begin{abstract}
Dental anxiety and phobia not rarely cause the patient to delay seeking oral health care if he ever seeks it at all. The present research aimed at providing a literature overview concerning to the applicability of Behavioral Medicine resources in management of dental anxiety and phobia, considering patients who have restrictions on use of drugs. Several risks factors are involved in the rise of such behaviors. These include educational, psychosocial, and economic factors. Comorbidity presentation can also occur, and the level of triggering elements can vary. Although the number of individuals affected by the psychological disorders is significant, the applicability and effectiveness of Behavioral Medicine resources as a means of their management are mostly reported in anecdotal studies. Due to the significant percentage of the society that is affected by dental anxiety and phobia, it produces a negative impact on both individual health and public health system, as shown in our findings. It becomes clear the need for developing new methodological approaches to the training of a more adequate number of professional able to deliver safe and effective Behavioral Medicine resources in medical care to patients with of dental anxiety and phobia, and thus lead to the better outcomes than has been observed.
\end{abstract}

Keywords: Anxiety; Phobia; Fear; Oral health; Behavioral medicine; Hypnosis

\section{Background}

Dentistry is a profession that has been improving a lot in the last years. Annually, new products and techniques are launched for dental improvement or care, increasingly following the aesthetic and functional needs of patients. As well as, globally, several educational institutions have been offered to professionals a wide variety of courses in new areas of dentistry with the goal of increasing patient care, efficient, effective and safe, since there is still a large percentage of the world's population that does not perform or avoid dental treatments due many reasons, including anxiety and dental phobia. According to Gordan et al. [1], dental anxiety and dental fear are factors that are independent of social, financial or educational status, affecting people of any age, gender or race. They cause the postponement or escape of dental treatment; consequently, they cause damage to physical and emotional health, sleep disturbance, increased use of medications and tendency to somatization. In addition, they cause social and financial damage in the work and school environments, and important implications for public health. There are several causes presented for dental anxiety and dental phobia, namely: painful or traumatic experiences in the dental environment in previous treatments; fear of pain; embarrassment for fear of negative social evaluation by the professionals, due to the state of teeth conservation or perceived negligence; feeling of helplessness associated with the distrust of professionals and their negative behaviors; co-morbid embarrassment and psychosocial dysfunction due to effects of sexual abuse, general anxiety, choking, fainting, panic attacks; looking for emergency or urgent care with the possibility of unfavorable outcomes; fear of needles; It is a fantasy about the image of the dentistry setting as frightening, absorbed through continuous verbal excitement conditioned in the family environment since childhood.

In the case of intervention in human beings, it is essential for the active dental surgeon to have a broad knowledge of human physiology and its needs, since the human being is a dynamic being, with a complex and changeable physiology throughout life, with multifactorial causes. Organic reactions from dental fear and anxiety may be associated with behavioral, cognitive, motor and 
physiological factors. The physiological responses resulting from dental anxiety and dental fear can in some cases be satisfactorily managed by healthy patients. However, patients with comorbidities may have a reduced tolerance level and cause homeostasis imbalance, compromising dental treatment. Monnazzi et al. [2], report that about $75 \%$ of medical emergencies in dental care are due to fear, anxiety and stress.

There are publications regarding the use of drugs to control dental anxiety and fear, with precise and safe indications. In Brazil the use of drugs for this purpose is made "per os via" or by inhalation. "Per os via", benzodiazepines are often administered for this purpose. By inhalation, proceed by inhalation of a mixture of nitrous oxide and oxygen. However, there are other resources and strategies that can be used to control stress and dental phobia. We then sought to describe a set of efficient and relatively safe tools for this purpose. We aimed to map the applicability of integrative medicine (mind/body) resources for the management of patients with dental anxiety and dental phobia.

\section{Development}

Dentistry is among the professions that contribute to the promotion of health individually and collectively, but it is found that the individual does not always access dental services under full conditions to maintain their satisfactory oral health throughout their lives. Perhaps the most common fear is the fear of pain. According to Badra [3] "Although we live in a time when pain can be controlled through remarkable drugs, the fear of pain still hangs over us as a constant threat." The National Institutes of Health (NIH) of the US National Library of Medicine defines fear as a primary emotion that indicates that a danger situation has been recognized, causing the individual to focus all his or her attention on the event, leading it into a situation. fight or flight, that is, it is an effective response to a current external danger that disappears with the elimination of the risk condition (MeSH/NIH). The NIH defines anxiety as the fear that has been transferred from the original situation to an imagined situation, arising from similar facts or triggering the recall of a previous situation. It also defines the feeling or emotion of fear, apprehension and impending disaster as non-disabling (MeSH/ $\mathrm{NIH}$ ). According to the NIH, the MeSH Terms, for dental anxiety and dental phobia are not distinguished, both of which are described as synonyms. Therefore, dental phobia or anxiety can be defined as abnormal fear or fear of visiting the dentistry setting for preventive care or therapy, and unjustified anxiety about dental procedures. We emphasize that in the literature articles were found that cite only one of the descriptors, or both simultaneously, according to the validated scales used in the studies. According to the Statistical Diagnostic Manual of Mental Disorders, Fifth Edition, (DSM 5) anxiety disorders are divided into twelve categories, and within these twelve categories the specific phobia is classified. Specific phobia is characterized by clinically significant anxiety caused by exposure to a dreaded situation or object, which often leads to avoidant behavior. Dental phobia is one of the subtypes of specific phobia. This subtype has a high familial aggregation and is often characterized by a strong vagal vessel response. Diagnostic criteria for Specific Phobia. DSMV, are:

a) Sharp fear or anxiety about an object or situation (e.g., injecting, seeing blood).

b) The phobic object or situation almost invariably elicits an immediate response of fear or anxiety.

c) The phobic object or situation is actively avoided or endured with intense anxiety or suffering.

d) Fear or anxiety is disproportionate to the real danger posed by the phobic object or situation and the cultural context.

e) Fear, anxiety or avoidance is persistent, usually lasting at least six months.

f) Fear, anxiety or avoidance causes clinically significant distress or impairment in social, professional or other important areas of life; AMERICAN PSYCHIATRIC ASSOCIATION, [4].

The DSM-5 recognizes that it is common for individuals to have multiple specific phobias, and generally fear three objects or situations. Patients with dental phobia usually have the following types of specific phobia, numbered as follows according to DSM-5.

(F40.230) Fear of blood

(F40.231) Fear of injections and transfusions

(F40.232) Fear of other medical care

(F40.233) Fear of injury (AMERICAN PSYCHIATRIC ASSOCIATION, [4].

The International Code of Diseases, ICD-10, describes as numbered (300.29) Phobic-Anxiety Disorders:

A group of disorders in which anxiety is evoked only or predominantly in certain well-defined situations that are not correctly dangerous. As a result, these situations are characteristically avoided or endured with dread. Patient concern may be focused on individual symptoms such as palpitations or fainting and is often associated with secondary fears of dying, losing control, or going crazy. The prospect of entering the phobic situation usually generates anticipatory anxiety. AMERICAN PSYCHIATRIC ASSOCIATION, [4]. And this group is subdivided into subgroups, and dental phobia falls into subgroup F40.2 Specific Phobias (isolated): "Phobias restricted to highly specific situations, such as proximity to certain animals, height, thunder, darkness, dentists or the sight of blood or injury. ."DSM 5, [4]. In the face of an anxious patient, it is necessary to recognize if symptoms of state or trait anxiety are presented, where the anxiety state is conceptualized by a transient state or condition of the human organism characterized by unpleasant feelings of tension and apprehension, consciously perceived with the increase. in the autonomic nervous system activity and anxiety trait, refers to differences in the tendency to react to situations perceived as threatening with intensified anxiety state [5]. According to Kaplan, Sadock \& Grebb [6], the peripheral manifestations of anxiety are diarrhea, dizziness, hypertension, 
hyperhidrosis, hyper-reflexia, palpitations, pupillary mydriasis, restlessness, syncope, tachycardia, tingling in the extremities, abdominal discomfort, frequency, retention and urinary urgency. Anxiety Disorders due to a General Medical Condition are due to systemic diseases. Symptoms are significant and are due to direct physiological effects of the medical condition and should be considered. Some general medical conditions associated with Anxiety, according to Kaplan, Sadock \& Grebb [6], are:

a) Primary diseases of the central nervous system: brain neoplasms; brain trauma and post-concussion syndrome; cerebrovascular disease; subarachnoid hemorrhage; migraine; encephalitis; cerebral syphilis; multiple sclerosis; Wilson's disease; Huntington's disease; epilepsy.

b) Systemic Conditions: heart disease. endocrine and inflammatory disorders, vitamin deficiencies.

c) Miscellaneous Conditions: hypoglycemia; carcinoid syndrome; systemic malignant conditions; premenstrual syndrome; febrile illnesses and chronic infections; porphyria; infectious mononucleosis; post hepatitis syndrome; uremia.

d) Toxic Conditions: abstinence from alcohol and drugs; amphetamines; sympathomimetic agents; vasopressor agents; caffeine and caffeine abstinence; penicillin; sulfonamides; cannabis; Mercury; arsenic; phosphor; organophosphates; carbon disulfide; benzene; aspirin intolerance.

e) Idiopathic Psychiatric Disorders: depression; mania; schizophrenia; anxiety disorders; generalized anxiety; panic attacks; phobic disorders; posttraumatic stress disorder.

Dental anxiety can overlap with an anxious picture that the patient already has and may worsen the picture itself or the clinical condition that the patient already had. It should be considered that even in dentistry setting there are conditions in which affected patients may present with comorbidity generalized anxiety as is the case of Burning Mouth Syndrome. Galli et al. [7]. Deva et al. [8], indicate the objective assessment by measuring blood pressure, heart rate, pulse oximetry and galvanic skin response, which may be momentarily altered allowing to categorize different levels of anxious states. Gordan et al. [1], further states that dental anxiety is characterized by a vicious circle in which retained or avoided individuals or dental treatment and consequently have dental problems that lead to avoiding treatment again; emergencies, reduce dental anxiety. In this circle are involved feelings of guilt, shame and inferiority, leading patients again to increased dental anxiety and avoid treatment. Morse and Chow [9], state that the most anxious moment is the application of anesthesia. And Heaton et al. [10], state that one in four adults report a clinically significant fear of dental injection, leading many to avoid dental treatment. Dental treatment is often a stressful experience for both the dentist and the patient, says Ruysschaert [11]. Deva et al. [8], note that treating these anxious patients is stressful for the dentist due to reduced cooperation, requiring more time and treatment resources, resulting in an unpleasant experience for both the patient and the dentist. In the first contact with the patient, the dental professional may observe some visible signs of patient anxiety. At every beginning of treatment an anamnesis is always necessary. Monson [12], states that the information obtained from a broad survey of the patient's history, implied the adoption of the best possible conduct to be adopted by the professional in relation to the planning of clinical procedures to be undertaken, and among them, they must necessarily be observed the degree of phobia and dental anxiety evidenced in the clinical presentation.

\section{Discussion}

Among the capabilities of Behavioral Medicine resources to minimize dental anxiety and dental phobia, is Hypnosis. Hypnosis is a regulated resource for dentistry, in Brazil, by Federal Government Law 5081, since August 24, 1966. Hypnosis in dentistry can be used as single or as co-intervention, with other Behavioral Medicine resources, such as Cognitive Behavioral Therapy (CBT) modalities, and in association to the chemical conventional anesthesia, with great indication, especially for those patients who have allergies to anesthetic components and as a complementary or substitute use of analgesic. Currently with the development of Contemporary Neuroscience, a vision is guided that allows the Dentistry professionals have access to knowledge of Behavioral Sciences, particularly linked to psychic diseases, where the emotional conditions in this paradigm have psychic and physical repercussions at the dentistry setting. The most striking example is the bruxism episode, which characterizes the gnashing of teeth. Thereafter there are outcomes such as tooth fracture, pulp necrosis, tooth loss, and temporomandibular joint disorders. The technological development, particularly neuroimaging studies, modern neuroscience studies have emerged. It is observed that the field of Hypnosis research has evolved much more. For this reason, Hypnosis is currently contextualized within Behavioral Medicine and is recognized as a validated therapeutic resource. Initially, according $\mathrm{NCCIH} / \mathrm{NIH}$, Hypnosis as one of the mind-body practices. In Dentistry was originally intended for anesthesia control. With the development of neuroscience itself, it is observed that the neuropsychic aspects linked to the lower third of the face transcend much more than was originally established. According to Gonçalves A. et al. [13], for a stimulus to be able to elicit a detectable or undetectable psycho-neuro-immuno-endocrinological response, it is necessary that the stimulus (information) be evaluated in at least three brain nuclei for interpreting the sense of reality, namely:

a) "Reptilian "nucleus": located on the bridge, evaluates the intensity (strong / weak). It is part of the Reptilian Brain described by Paul Maclean in 1970;

b) Limbic nucleus: located in the limbic amygdala, assesses whether the consciously perceived stimulus was good or bad, i.e. the quality (pleasant or unpleasant);

c) Neocortical nucleus evaluates the impairment of the integrity of the individual from the results of the nuclei evaluations previously mentioned. From the perception of these stimuli 
arise the behavioral responses of coping, dodging, attacks or freezing.

In humans, the psycho-neuroimmune endocrine axis (PNIE), conscious or not, presents a rationally aggregated response where a sophisticated system of social behavioral rules generically called Moral Profit Investments, Wright [14], most often influence and are systematically considered by the individual in deciding the type of behavioral response to be exuberant. Martins MB et al. [15], proposes a welcoming action which requires an intention of transformation, with the purpose of education that can intentionally resignify the care, which leads to a reflection on the need to have an insight into the problems that go beyond the biological aspects of the applicability of complementary therapies. Several studies are presented in the literature with mind-body medicine resources to reduce dental fear and anxiety. Hypnosis is a psycho-physical state of greater receptivity to suggestion and is usually conducted by the hypnotist (MeSH Terms). According to Hammond [16] a huge volume of research provides convincing evidence that hypnosis is an effective treatment for state anxiety, well used in surgeries and medical and dental procedures. Kleinhauz \& Eli [17] state that the use of Hypnosis is well indicated in patients who are hypersensitive to local anesthetics. Abdeshahi et al. [18] argue that the use of hypnosis prior to surgical dental treatment has been shown to reduce anxiety, pain and hemorrhage and to reduce postoperative analgesic use. Huet et al. [19] attest that hypnosis can be effective in reducing anxiety and pain in children who have received dental anesthesia. Lu [20] reports the Hypnosis can reduce the amount of sedative that would be needed to alleviate anxiety in geriatric patients; with heart or kidney failure; liver disease, and those with other severe systemic conditions that need to undergo dental procedures, as in these cases the use of sedatives may increase the risk of adverse events for these patient groups.

Hammond [16] suggests that Self-Hypnosis training represents a quick and inexpensive alternative and proves to be safe for treating anxiety-related conditions. According to Deva et al. [8] Hypnosis has significant positive effects on anxiety, pain, behavior and physiological parameters when compared to the absence of treatment. Juge \& Tubert Jeannin [21] state that individualized hypnosis brings more benefits than standardized hypnosis with audio recordings. AL Harasi et al. [22] believe that there is insufficient evidence of benefits to justify the use of Hypnosis in Paediatric Dentistry. Imaging consists in making use of mental imagery produced by the imagination as a form of psychotherapy, and is often part of Hypnosis, Relaxation Techniques and other Behavioral Medicine therapies modalities (MeSH). According to Armitage and Reidy [23] the Imagery used as a simulation process has been used to reduce anxiety and is described as more effective than the use of simulation alone, where Imagery consists only of the picture of the situation. performed, and is still considered a brief and self-directed intervention.

Desensitization is a Behavioral Medicne therapeu modality in which deep muscle relaxation is used to inhibit the effects of graduated anxiety stimuli (MeSH Terms). According to Heaton et al. [10] and Moore et al. [24] systematic desensitization has been shown to be a more common therapeutic method for treating specific phobias, such as fear of dental injections. Taylor and Campbell, state that the needle desensitization technique has been effective in pediatric patients. Biofeedback is a therapy that uses a device that provides the status of the individual's autonomic nervous system function (e.g. skin temperature, heartbeat, brainwave), such as visual or auditory feedback in order to provide conditions related to self-control (e.g. hypertension, migraines) (MeSH Tems). Deva et al. [8] report that the information provided by this technique assists the patient in obtaining self-regulation of the physiological process being monitored.

Cognitive therapy consists of a direct form of psychotherapy based on the interpretation of situations (cognitive structure of experiences) that determine how an individual feel and behaves. It is based on the premise that cognition, the process of knowledge acquisition and belief formation, is a major determinant of mood and behavior. According to Deva et al. [8], Anxious patients most often have inadequate expectations and beliefs about dental treatment. Modifying such negative cognitions is a means of reducing anxiety. It is noteworthy that cognitive behavioral therapy is a combination of behavioral therapy, a therapy where the application of modern learning and conditioning theories in the treatment of behavioral disorders (MeSH Terms) and cognitive therapy occurs. Deva et al. [8], state that this is the most accepted and successful psychological treatment for dental anxiety and phobia. Oakley et al. [25] propose the use of cognitive behavioral therapy in temporo-mandibular joint (TMJ) dysfunctions, including relaxation training, self-monitoring of stressors, and cognitive coping strategies, demonstrated that treatment had its greatest impact on improving mood, especially anxiety, and noted that there were some effects on patients' pain experiences.

According to Colas, [26], in clinical practices to deal with anxiety states in general, organize the so-called cognitive behavioral analysis (Figure 1), where one can identify the activating Dentistry professionals event and the behavioral scheme composed by the triad. : avoidance attitude, physiological reactions to clinical signs of anxiety - tachycardia, sweating, irregular breathing and finally the feelings of fear, anger, sadness, guilt related to the attitude manifested in this scheme. The Behavioral Scheme is immediately or concomitantly self-rated by the cognitive scheme which is: automatic thinking "I will not succeed" and dysfunctional belief "I am unable or weak". This scheme provides indications of how belief and value structures deal with their identity and ability problems. Colas, [26]. Another point of cognitive behavioral analysis is related to the so-called cognitive structures, which involve the organic and biological part of each individual, the conditions and perception of pain, inflammatory reactions, tolerance to oral manipulation, the belief system and values of each individual, identity values, social, cultural and ultimately, life history as personal trauma with previous treatments, or even imaginary experiences - told by other individuals. Cognitive structures lead to distortions of cognitive processing through generalizations, omissions, and distortions of information. Colas, [26] (Figure 1). 


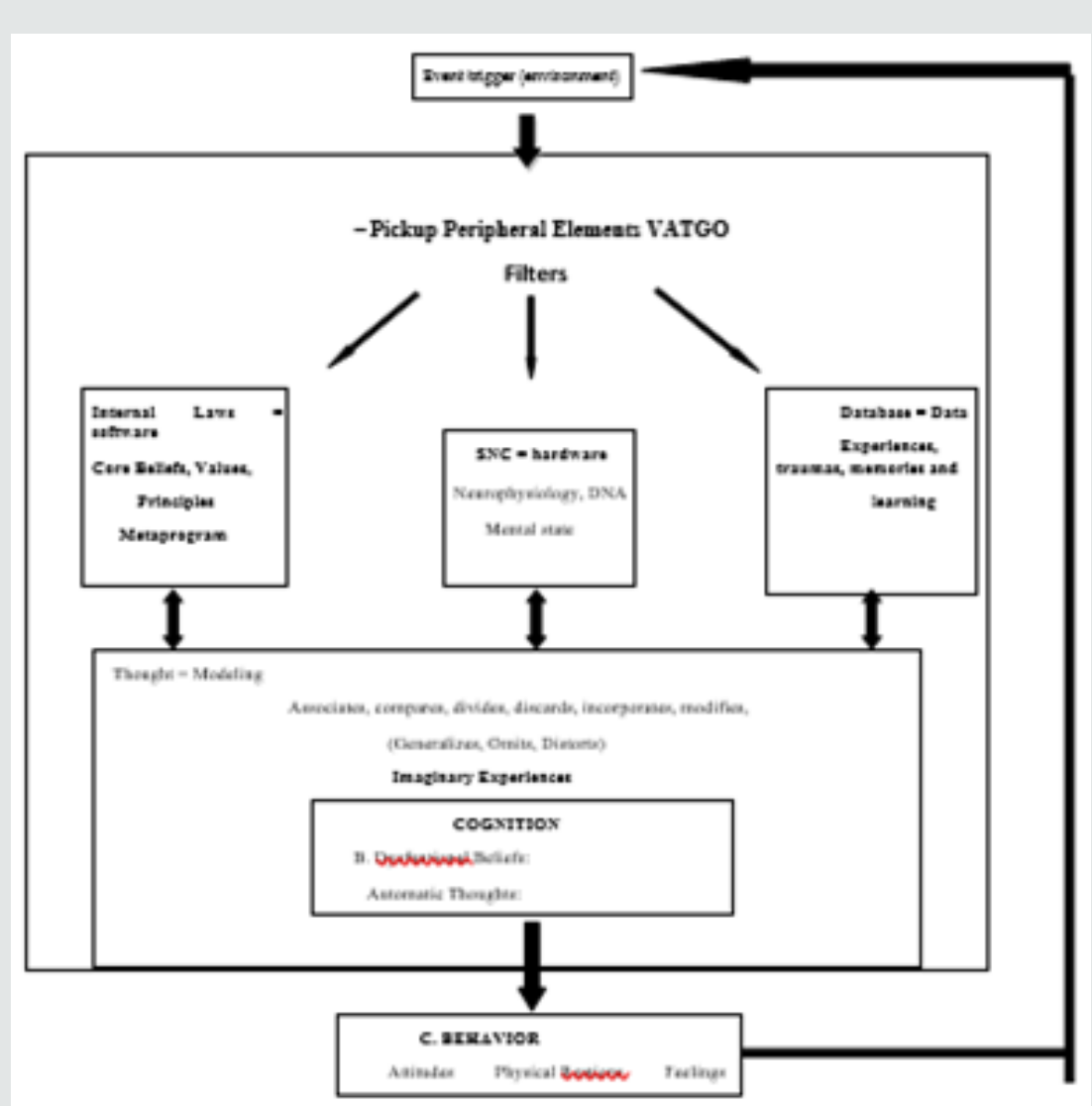

Figure 1: Cognitive Behavior Model.

\section{Examples of feelings in a single word}

Depressed Anxious Angry Guilty Ashamed Sad Embarrassed Excited Terrified Angry Insecure Proud Angry Nervous Panicking Frustrated Bored Hurt Angry Scared Happy Loving Humiliated Unable Wretched Inappropriate. As we see, a detailed investigation of this cognitive scheme is necessary to program the focus of action. There are several possibilities for practicing Hypnosis in association with Behavioral Medicine practices. In the behavioral scheme, the reason for the patient's arrival, we can act with psychoeducation by interpreting the entire scheme of the patient's cognitive behavioral analysis and explaining the initial need to learn to manage their stress through breathing and relaxation techniques as mentioned earlier. Meditation, mindfulness, and self-hypnosis techniques are fundamental to better stress management, if time permits. This simple intervention plays an important role in managing the triad: muscle tension "relaxation" - neuro-vegetative tension "inhibition of the sympathetic system" - and psycho-emotional tension in decreasing stress, anxiety, increasing tolerance, and decreasing criticism. We can interfere with the cognitive scheme by making a Socratic debate under relaxation where logical reasoning is relaxed and suggestions are more easily accepted, acting upon cognitive structures, acting on the core belief and value system, using roleplaying, assertiveness, modeling techniques. etc., thereby easing seemingly inflexible beliefs; It can play a role in life history, using "regression" cognitive rescue techniques, where it weakens previous traumas or even re-signifies them with specific techniques. Finally, acting directly on the individual's imaginary experience, inducing a local anesthetic and subsequently dissociating the patient, leading "one part of his mind" to a pleasant memory while the "other part of the mind" is anesthetized in the dentist's chair.

However, we need to make it clear that the two most important points of this approach are: firstly an intense rapport that must be developed by the dentist and the patient; and the second point is psychoeducation, where the use of the attached scheme is an interesting facilitating tool for the patient to understand and validate the possibilities of interventions. Relaxation Therapy is a treatment to improve health, using techniques that can reduce physiological stress, psychological stress, or both (MeSH Terms). According to Armfield and Heaton [10,27] and Rayman et al. [28] the use of behavioral techniques alone help induces patient relaxation, reduce anxiety and may reduce the need for analgesics as well as their side effects. Deva et al. [8] cite various relaxation techniques, such as Ost relaxation technique, Jacobsen progressive muscle relaxation, functional relaxation, rapid relaxation technique, and autogenous relaxation de Schultz Biggs et al. [29] state that it is preferable to use relaxation techniques that are already known to the patient if he practices them. Yoga is a great orthodox system of Hindu philosophy based on Sankhya (metaphysical dualism) but 
differs from it in being theistic and characterized by the teaching of raja yoga as a practical method of liberating the self. It includes an exercise system to achieve bodily or mental control and wellbeing with self-liberation and union with the universal spirit (MeSH terms). Shankarapillai et al. [30] conducted a study where yogi breathing was applied to a group of students who underwent their first periodontal surgery. The authors observed a decrease in anxiety level compared to the control group.

Aromatherapy is the use of perfumes and plant essences that can affect or change a person's mood or behavior and facilitate physical, mental and emotional well-being. Chemicals consisting of plant essential oils have a number of therapeutic properties and have been used historically in Africa, Asia and India. Its major application is in the field of alternative medicine (MeSH terms). Kritsidima et al. [31] found that the scent of lavender oil reduces the anxiety level of patients waiting to be seen at a previously scheduled dental appointment when compared to the control group. Faced with various therapeutic modalities of Behavioral Medicine available to minimize dental anxiety and dental phobia, Deva et al. [8], state that therapy should be tailored to each individual following an appropriate assessment, and should be based on the dentist's experience, expertise, degree of anxiety, patient's intellect, age, cooperation, and clinical situation presented. Behavioral Medicine practices are effective on a long-term basis with positive effects on patients, enabling them to seek dental care in the future, which should be the primary focus of the dental team. Mildly and moderately anxious patients can be managed using psychological interventions.

The advantages gained through Behavioral Medicine practices along with an understanding of psychology in the management of dental anxiety, suggest that the use of non-pharmacological techniques for controlling dental anxiety may maximize adherence to the ethical principles of patient beneficence and autonomy. However, according to Donate Bartfield [32], the applicability of non-pharmacological interventions for anxiety management is difficult for dentists to routinely use, and it is suggested that further training in these specialties and increased integration with health professionals. Mental disorders are necessary for dentists. The present study, is the synthesis of qualitative systematic review, based on electronic search in Medline databases, via PubMed and Central (Cochrane Library) and manual search in congress annals, books, magazines on the theme "applicability of mind-body medicine resources in the care of patients with dental anxiety and dental phobia". The Medical Subject Headings used were dental anxiety, dental phobia, mind-body practices, behavioral medicine, associated in a PICO question - What is the applicability of behavioral medicine resources to the management of dental anxiety and phobia? Several studies have been found on cases of dental anxiety and dental phobia and different forms of management through Behavioral Medicine practices pharmacological, resources and by combining two or more resources. The literature, however, emphasizes the importance of an accurate diagnosis that will assist the clinician in the best choice of treatment. Studies have shown that the aetiology of dental anxiety and dental phobia is multifactorial, and therefore, according to Deva et al. [8], there is no single therapy able for its management. Adequate assessment of anxiety level is required during care to allow the Dentistry professionals to perform a proper treatment plan.

Vinckier \& Vansteenkiste [33] state that before thinking about the best way to treat a patient with dental anxiety, it is necessary to emphasize preventive dentistry. Lipp et al. [34] and Jonhson et al. [35] suggest that basic information given about treatment decreases the level of dental anxiety, while near-zero or very prolonged information increases the level of anxiety. Although emphasizing the needs for differentiated attention to identify patients with anxiety and dental phobia, and to mitigate the obstacles that prevent them from performing dental treatments in order to allow them to achieve quality of oral health and general well-being, it is observed. In addition to expressing the principle of benevolence, the dental surgeon needs specialized knowledge in the management of these patients with regard to biopsychosocial aspects and for this, the continuing education of dental professionals in matters pertaining to Behavioral Medicine is necessary [36-38].

\section{Final Considerations}

At first, the aim the present study was to search in the literature alternatives for the management of patients with dental anxiety and dental phobia through Behavioral Medicine resources. Despite the evidence levels on the subject still lack studies of better methodological quality, after searching for a theoretical basis in relation to the history of symptoms, it was possible to map several studies that show the effectiveness of some mind-body medicine resources for the management of patients with dental anxiety and dental phobia. the effectiveness and safety of such resources. For this study, it is evident that psychological symptoms and quality of life improve in response to treatment with co-intervention of Behavioral Medicine resources, showing a decrease in dental anxiety levels, patients are more receptive to dental treatment breaking the vicious circle of avoidance of treatment and consequent damage to oral and general health. Improvement of self-esteem and self-confidence is also observed, with the motivation of frequent and continuous self-care. Dental anxiety and dental phobia have multifactorial causes, with or without comorbidities, and diversified levels, involving educational, psychosocial and economic factors. Due to the significant portion of society that is affected by these disorders, impacting on individual health and the public health system, as shown in the findings, a methodological approach is needed to train more skilled professionals. to dispense Behavioral Medicine resources care that is safe and effective in treating people suffering from dental anxiety and phobia.

\section{References}

1. Gordon D, Heimberg RG, Tellez M, Ismail A (2013) A critical review of approaches to the treatment of dental anxiety in adults. J Anxiety Disord (4): $365-378$.

2. Monnazzi (1993) Emergências e Urgências Médicas. Como proceder? Revista Gaucha de Odontologia 49(1): 7- 11. 
3. Badra Álvaro (1987) Hipnose em Odontologia e Odontologia Psicossomática. Andrei, São Paulo, Brazil.

4. Porto Alegre Artmed (2014) Manual Diagnóstico e Estatístico de Transtornos Mentais V - $5^{\circ}$ Edição -American Psychiatric Association pp. 197-198.

5. Gorestein L, HSG, Zuardi AW (2000) Escala de Avaliação Clínica em Psiquiatria e Psicofarmacologia. São Paulo: Lemos Editorial pp. 140.

6. Kaplan HI, Sadock BJ, Grebb JA (1997) Compêndio de Psiquiatria Ciências do Comportamento e Psiquiatria Clínica. 7o edição. Porto Alegre: Artes Médicas pp. 549-550.

7. Federica Galli, Giovanni Lodi, Andrea Sardella, Elena Vegni (2017) Role of psychological factors in burning mouth syndrome: A systematic review and meta-analysis. Cephalalgia, International Headache Society 37(3): 265-277.

8. Deva, Priya Appukuttan (2016) Strategies to manage patients with dental anxiety and dental phobia: literature review. Clin Cosmet Investig Dent 8: 35-50.

9. Morse DR, Chow E (1993) The effect of the Relaxodont brain wave synchronizer on endodontic anxiety: evaluation by galvanic skin resistance, pulse rate, physical reactions, and questionnaire responses. Int J Psychosom 40(1-4): 68-76.

10. Heaton LJ, Leroux BG, Ruff PA, Coldwell SE (2013) Computerized dental injection fear treatment: a randomized clinical trial. J Dent Res 92(7 Suppl): 37-42.

11. Ruysschaert N (2003) Hypnosis and hypnotic phenomenon. Preliminary aid in cases of stress for the patient and dentist. Rev Belge Med Dent 58(2): 105-117.

12. Monson CA, Monson ASC (2019) Terapias Complementares para o Paciente Crônico na Perspectiva da Saúde Baseada em Evidências. 1. Edition. Apris, Curitiba.

13. Gonçalves A, Damasceno D (2016) Um Olhar Novo na Saúde Oral Aspectos psicodinâmicos do atendimento odontológico. São Paulo.

14. Wright R (1995) The Moral Animal. Blackwell Editors: New York, USA.

15. Martins MB (2018) Acolhimento Terapêutico na psoríase São Paulo.

16. Hammond DC (2010) Hypnosis in the treatment of anxiety- and stressrelated disorders. Expert Rev Neurother (2): 263-273.

17. Kleinhauz M, Eli I (1993) When pharmacologic anesthesia is precluded: the value of hypnosis as a sole anesthetic agent in dentistry. Spec Care Dentist 13(1): 15-18.

18. Abdeshahi SK, Hashemipour MA, Mesgarzadeh V, Shahidi PA, Halaj MA (2013) Effect of hypnosis on induction of local anaesthesia, pain perception, control of haemorrhage andanxiety during extraction of third molars: a case-control study. J Craniomaxillofac Surg 41(4): 310315.

19. Huet A, Luca S Polomei MM, Robert JC, Sixou JL, Wodey E (2011) Hypnosis and dental anesthesia in children: a prospective controlled study. Int J Clin Exp Hypn 59(4): 424-440.

20. Lu DP (1996) Hypnosis and pharmacological sedation for medicaly compromised patients. Compend Contin Educ Dent 17(1): 32, 34-36,3840.
21. Jugé C, Tubert Jeannin S (2013) Effects of hypnosis in dental care. Presse Med 42(4Pt 1): 114-124.

22. AL Harasi S, Asheley PF, Moles DR, Parekh S, Walters V (2010) Hypnosis for children undergoing dental treatment. Cochrane Database Syst Rev 8: CD007154.

23. Armitage CJ, Reidy JG (2012) Evidence that process simulations reduce anxiety in patients receiving dental treatment: randomized exploratory trial. Anxiety Stress Coping 25(2): 155-165.

24. Moore R, Abrahamsen R, Brodsgaard I (1996) Hypnosis compared with group therapy and individual desensitization for dental anxiety. Eur Oral Sci 104(5-6): 612-618.

25. Oakley ME, McCrerary CP, Clark GT, Holston S, Glover D, et al. (1994) A cognitive-behavioral approach to temporomandibular dysfunction treatment failures: a controlled comparison. J Orofac Pain Fall 8(4): 397401.

26. Colás OC (2012) Psicoeducação em Hipnoterapia CognitivoComportamental: in Temas de Hipnologia 185-192.

27. Armfield JM, Heaton LJ (2013) Management of fear and anxiety in the dental clinic: a review. Aust Dent J 58(4): 390-407.

28. Rayman S, Dincer E, Almas K (2013) Managing dental fear and anxiety. N Y State Dent J 79(6): 25-29.

29. Biggs QM, Kelly KS, Toney JD (2003) The effects of deep diaphragmatic breathing and focused attention on dental anxiety in a private practice setting. J Dent Hyg 77(2): 105-113.

30. Shankarapillai R, Nair MA, George R (2012) The effect of yoga in stress reduction for dental students performing their first periodontal surgery: A randomized controlled study. Int J Yoga 5(1): 48-51.

31. Kritsidima M, Newton T, Asimakopoulou K (2010) The effects of lavender scent on dental patient anxiety levels: a cluster randomised-controlled trial. Community Dent Oral Epidemiol 38(1): 83-87.

32. Donate Bartfield E, Spellecy R, Shane NJ (2010) Maximizing beneficence and autonomy. Ethical support for the use of nonpharmacological methods for managing dental anxiety. J Am Coll Dent Fall 77(3): 26-34.

33. Vinckier F, Vansteenkist EG (2003) A strategy for treating the anxious patient. Rev Belga Med Dent 58(4): 209-220.

34. Lipp M, Dick W, Daublander M, Bertram M (1991) Different information patterns and their influence on patient anxiety prior to dental local anesthesia. Dtsch Z Mund Kiefer Gesichtschir 15(6): 449457.

35. Johnson S, Chapman K, Huebner G (1984) Stress reduction prior to oral surgery Anesth Prog 31(4): 165-169.

36. Porto Alegre: Artmed (2014) Manual Diagnóstico e Estatístico de Transtornos Mentais V - $5^{\circ}$ Edição - American Psychiatric Association pp. 197-198.

37.www.planalto.gov.br/ccivil_03/leis/L5081.htm

38. http://www.ncbi.nlm.nih.gov/pubmed 
(C) This work is licensed under Creative

To Submit Your Article Click Here:

Submit Article

DOI: $10.32474 /$ GJAPM.2019.02.000129

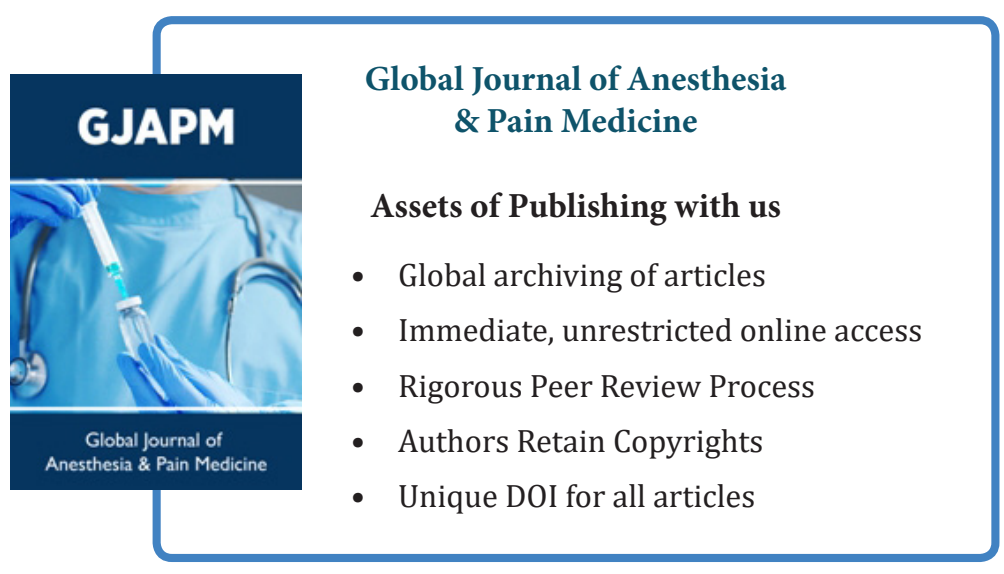

